\title{
Maximal Levi subgroups acting on the Euclidean building of $\mathrm{GL}_{n}(F)$
}

\author{
Jonathan Needleman
}

full screen

close

\section{Abstract}

In this paper we give a complete invariant of the action of $\mathrm{GL}_{n}(F) \times$ $\mathrm{GL}_{m}(F)$ on the Euclidean building $\mathcal{B}_{e} \mathrm{GL}_{n+m}(F)$, where $F$ is a discrete valuation field. We then use this invariant to give a natural metric on the resulting quotient space. In the special case of the torus acting on the tree $\mathcal{B}_{e} \mathrm{GL}_{2}(F)$, we obtain an algorithm for calculating the distance of any vertex in the tree to any fixed apartment.

Keywords : affine building, Euclidean building, Levi subgroup, group action MSC 2000: 20E42, 20G25

\section{Introduction}

To understand distance in the 1-skeleton of a building $\mathcal{B} G$ associated to a reductive algebraic group $G$, one may look at a stabilizer $K$ of a point, and then study the action of $K$ on $\mathcal{B} G$. When working over a discrete valuation field vertices correspond to maximal compact subgroups. This analysis gives rise to information about $K \backslash G / K$, and therefore the Hecke algebra [4, 5].

In this paper we specialize to $G=\mathrm{GL}_{n}(F)$ and are interested in the double cosets $L \backslash G / K$, where $L \cong \mathrm{GL}_{n_{1}}(F) \times \mathrm{GL}_{n_{2}}(F)$ is a maximal Levi subgroup of $G$. The study of the action of $L$ on the building $\mathcal{B}_{e} \mathrm{GL}_{n}(F)$ will lead to a description of distance from any vertex to a certain subbuilding stabilized by $L$. In the case when $n=2$ and $L=T$ is a maximal split torus, our description gives a way of calculating the distance from a given point to a fixed apartment.

We also give a combinatorial description of the quotient space $L \backslash \mathcal{B}_{e} \mathrm{GL}_{n}(F)$ as follows. Let $A^{n}=\left\{\left(\alpha_{i}\right)_{i=1}^{n} \mid \alpha_{i} \in \mathbb{N}, \alpha_{i} \geq \alpha_{i+1}\right\}$. If $n_{1} \leq n_{2}$ there is an graph isometry between $L \backslash \mathcal{B}_{e} \mathrm{GL}_{n}(F)$ and $A^{n_{1}}$ where $A^{n}$ is endowed with the 


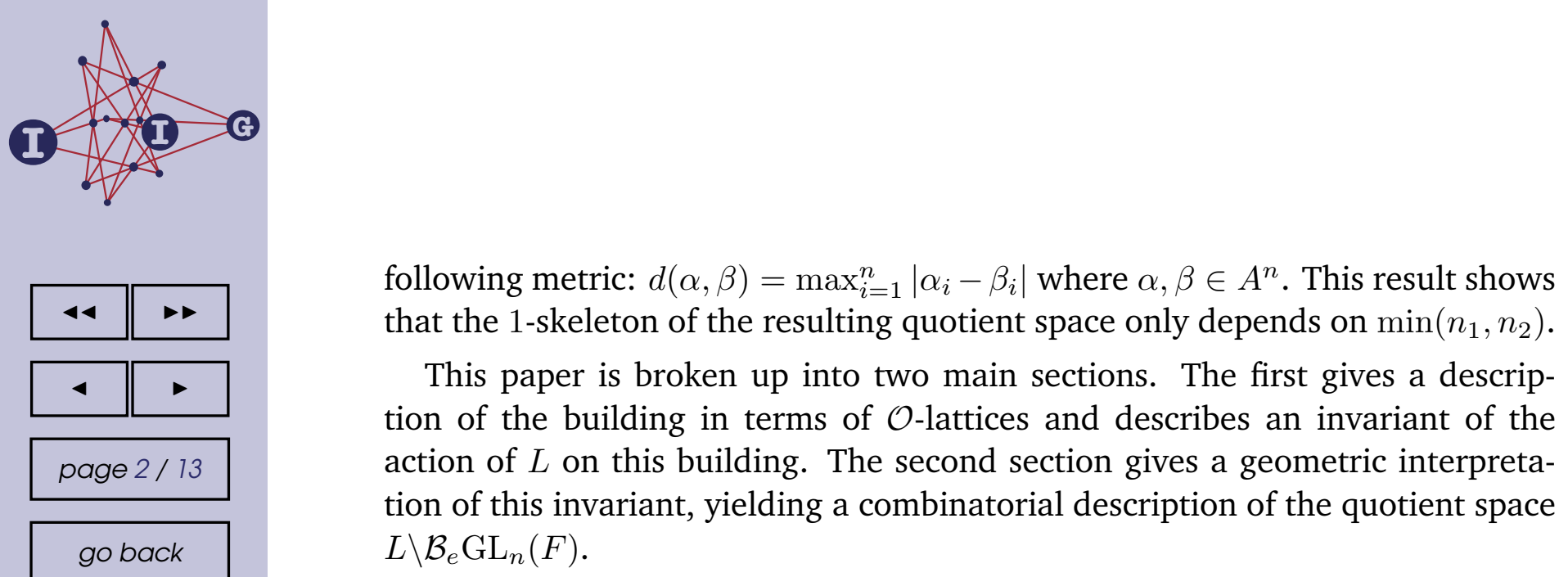

full screen

close

\section{Orbits of maximal Levi factors on $\mathcal{B}_{e} \mathrm{GL}(V)$}

\section{1. $\mathcal{O}$-lattices and $\mathcal{B}_{e} \mathrm{GL}(V)$}

Throughout this paper let $F$ be a discrete valuation field with valuation $v$. We will denote the ring of integers in $F$ by $\mathcal{O}$, and fix once and for all a uniformizer $\varpi$ of $\mathcal{O}$. Let the unique maximal prime ideal be denoted as $\mathcal{P}=(\varpi)$, and the residue field $\mathcal{O} / \mathcal{P}$ will be denoted by $\mathfrak{k}$. Let $\mathcal{P}^{k}=\left(\varpi^{k}\right)$ for $k \in \mathbb{Z}$. Then $\log _{\mathcal{P}}\left(\mathcal{P}^{k}\right)=k$. Let $V$ be a finite dimensional vector space defined over $F$ of dimension $n$. We will describe the Euclidean building $\mathcal{B}_{e} \mathrm{GL}(V)$ associated to $\mathrm{GL}(V)$. For more details see [1]. Let $\Lambda \subset V$ be a finitely generated free $\mathcal{O}$-module of rank $n$. Denote by $[\Lambda]$ the homothety class of $\Lambda$, that is $[\Lambda]=\left\{a \Lambda \mid a \in F^{\times}\right\}$.

Homothety classes of lattices will form the vertices of $\mathcal{B}_{e} \operatorname{GL}(V)$. Two vertices $\lambda_{1}, \lambda_{2} \in \mathcal{B}_{e} \mathrm{GL}(V)$ are incident if there are representatives $\Lambda_{i} \in \lambda_{i}$ so that $\varpi \Lambda_{1} \subset \Lambda_{2} \subset \Lambda_{1}$, i.e. $\Lambda_{2} / \varpi \Lambda_{1}$ is a k-subspace of $\Lambda_{1} / \varpi \Lambda_{1}$. The chambers in $\mathcal{B}_{e} \mathrm{GL}(V)$ are collections of maximally incident vertices. To put this more concretely, a chamber is a collection of $n$ vertices $\lambda_{0} \cdots \lambda_{n-1}$ with representatives $\Lambda_{0} \cdots \Lambda_{n-1}$ satisfying $\varpi \Lambda_{0} \subsetneq \Lambda_{1} \subsetneq \cdots \subsetneq \Lambda_{n-1} \subsetneq \Lambda_{0}$. A wall of a chamber is any subset of $n-1$ vertices in the given chamber. We will denote by $\mathcal{B}_{e} \mathrm{GL}(V)^{k}$ the set of all facets of $\mathcal{B}_{e} \mathrm{GL}(V)$ of dimension $k$.

A frame $\mathcal{F}$ in $V$ is a collection of lines $l_{1}, \ldots, l_{n} \subset V$ which are linearly independent and span all of $V$. We now describe certain subcomplexes of $\mathcal{B}_{e} \mathrm{GL}(V)$. Define $\mathcal{A}_{\mathcal{F}}$ to be the subcomplex consisting of vertices $[\Lambda]$ of the following form:

$$
\Lambda=\bigoplus_{i=1}^{n} \mathcal{O} e_{i}
$$

where $e_{i} \in l_{i} \in \mathcal{F}$. Then $\mathcal{A}_{\mathcal{F}}$ is an apartment of $\mathcal{B}_{e} \mathrm{GL}(V)$, and every apartment is uniquely determined by a frame in this way.

The group $\mathrm{GL}(V)$ has a natural action of $\mathcal{B}_{e} \mathrm{GL}(V)$, namely the one induced from the action of GL( $V)$ on $V$. This action preserves distance in the building. 


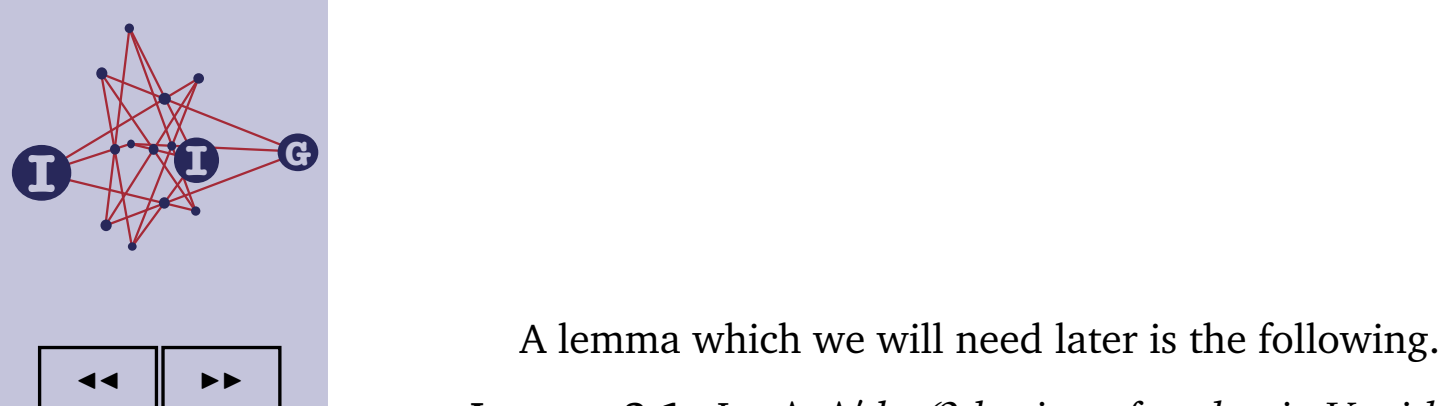

Lemma 2.1. Let $\Lambda, \Lambda^{\prime}$ be $\mathcal{O}$-lattices of rank $n$ in $V$ with $\Lambda^{\prime} \subset \Lambda$. Then the natural map from $\mathrm{GL}(\Lambda) \cap \operatorname{stab}\left(\Lambda^{\prime}\right)$ to $\mathrm{GL}\left(\Lambda / \Lambda^{\prime}\right)$ is surjective.

Proof. This result appears to be well known, but the proof could not be found in the literature and so is given here. There is an $\mathcal{O}$-basis $\left\{e_{1}, \ldots, e_{n}\right\}$ of $\Lambda$ so that $\left\{\varpi^{k_{1}} e_{1}, \ldots, \varpi^{k_{n}} e_{n}\right\}$ with $k_{i} \in \mathbb{N}$ is an $\mathcal{O}$-basis of $\Lambda^{\prime}$. This is equivalent to the statement that for any two vertices there is an apartment which contains them both. For $\bar{\sigma} \in \operatorname{GL}\left(\Lambda / \Lambda^{\prime}\right)$ we will construct $\sigma \in \operatorname{GL}(\Lambda) \cap \operatorname{stab}\left(\Lambda^{\prime}\right)$ which descends to $\bar{\sigma}$.

Let $\bar{e}_{i}$ be the image of $e_{i}$ in $\Lambda / \Lambda^{\prime}$. Then

$$
\bar{\sigma}\left(\bar{e}_{i}\right)=a_{1}^{i} \bar{e}_{1}+\cdots+a_{n}^{i} \bar{e}_{n}
$$

where $a_{j}^{i} \in \mathcal{O}$. Observe that $a_{j}^{i}$ is unique modulo $\mathcal{P}^{k_{j}}$. Then define $\sigma$ on the $\mathcal{O}$-basis $\left\{e_{1}, \ldots, e_{n}\right\}$ of $\Lambda$ as follows:

$$
\sigma\left(e_{i}\right)= \begin{cases}\sum_{j=1}^{n} a_{j}^{i} e_{j} & \text { if } \bar{e}_{i} \neq 0 \\ e_{i} & \text { if } \bar{e}_{i}=0\end{cases}
$$

What needs to be shown is that $\sigma$ is invertible and leaves $\Lambda^{\prime}$ invariant.

First, we show $\sigma$ leaves $\Lambda^{\prime}$ invariant.

$$
0=\bar{\sigma}\left(\varpi^{k_{i}} \bar{e}_{i}\right)=a_{1}^{i} \varpi^{k_{i}} \overline{e_{1}}+\cdots+a_{n}^{i} \varpi^{k_{i}} \bar{e}_{n}
$$

This shows that $a_{j}^{i} \varpi^{k_{i}} \in \mathcal{P}^{k_{j}}$, and so $\sigma\left(\varpi^{k_{i}} e_{i}\right) \in \Lambda^{\prime}$.

Next we show invertibility. Let $\sigma^{*}$ be the construction given above for $\bar{\sigma}^{-1}$, and let $\tau=\sigma \circ \sigma^{*}$. This will be a function which is a lift of the identity map in $\operatorname{GL}\left(\Lambda / \Lambda^{\prime}\right)$. Let $M=\operatorname{span}_{\mathcal{O}}\left\langle e_{i} \mid \bar{e}_{i} \neq 0\right\rangle$ and let $M^{\prime}=\operatorname{span}_{\mathcal{O}}\left\langle e_{i} \mid \bar{e}_{i}=0\right\rangle$. Then $\left.\tau\right|_{M}=\mathrm{id}+E$ where $E \in \operatorname{Hom}_{\mathcal{O}}\left(M, \Lambda^{\prime}\right)$ and is id on $M^{\prime}$. Any $\tau$ of this form is invertible and hence so is $\sigma$.

\section{2. $\mathrm{GL}\left(W_{1}\right) \times \mathrm{GL}\left(W_{2}\right)$ acting on $\mathcal{B}_{e}\left(\mathrm{GL}\left(W_{1} \oplus W_{2}\right)\right)$}

Let $V$ be a vector space over $F$. Fix a maximal Levi subgroup $L$ of $\operatorname{GL}(V)$. Associated to $L$ are subspaces $W_{1}, W_{2} \subset V$ satisfying $V=W_{1} \oplus W_{2}$. Then $L \cong \mathrm{GL}\left(W_{1}\right) \times \mathrm{GL}\left(W_{2}\right)$. In this section we will describe the orbits of the action of $\mathrm{GL}\left(W_{1}\right) \times \mathrm{GL}\left(W_{2}\right)$ on $\mathcal{B}_{e} \mathrm{GL}(V)^{0}$ in terms of an invariant $Q$. Additionally we will give a representative of each orbit.

Let $p_{i}$ be the projection of $V$ onto $W_{i}$ with respect to our given decomposition. We will use these maps to define invariants of the vertices and then show for our action that these invariants classify all orbits. 
Let $\Lambda$ be an $\mathcal{O}$-lattice. We make the following definitions for $i=1,2$ :

$$
\begin{aligned}
P_{i}(\Lambda) & =\operatorname{Im}\left(\left.p_{i}\right|_{\Lambda}\right), \\
K_{i}(\Lambda) & =\operatorname{Ker}\left(\left.p_{i^{\prime}}\right|_{\Lambda}\right)=\Lambda \cap W_{i},
\end{aligned}
$$

where $i^{\prime}=(i \bmod 2)+1$.

These are lattices in $W_{i}$.

Lemma 2.2. $K_{i}(\Lambda) \subset P_{i}(\Lambda)$.

Proof. If $v \in K_{i}(\Lambda)=\Lambda \cap W_{i}$, then $v \in \Lambda$, so $p_{i}(v) \in P_{i}(\Lambda)$. But $p_{i}(v)=v$ since $v \in W_{i}$.

By Lemma 2.2 we can define $Q_{i}(\Lambda)=P_{i}(\Lambda) / K_{i}(\Lambda)$. This is a finitely generated torsion $\mathcal{O}$-module.

Proposition 2.3. $Q_{1}(\Lambda) \cong Q_{2}(\Lambda)$ as $\mathcal{O}$-modules. This isomorphism class will be denoted by $Q(\Lambda)$.

Proof. We make slight modifications to the proof found in [2]. Let $p_{i}^{\prime}: \Lambda \rightarrow$ $Q_{i}(\Lambda)$ be the composition of $p_{i}$ with the natural projection map $\pi_{i}: P_{i}(\Lambda) \rightarrow$ $Q_{i}(\Lambda)$. We define a map so that for all $v \in \Lambda$

$$
\begin{aligned}
\Theta: Q_{1}(\Lambda) & \rightarrow Q_{2}(\Lambda) \\
p_{1}^{\prime}(v) & \mapsto p_{2}^{\prime}(v) .
\end{aligned}
$$

We will show that $\Theta$ is well defined, and is an isomorphism.

Let $w_{1}+w_{2}, w_{1}^{\prime}+w_{2}^{\prime} \in \Lambda$ with $w_{i}, w_{i}^{\prime} \in W_{i}$ and $\pi_{1}\left(w_{1}\right)=\pi_{1}\left(w_{1}^{\prime}\right)$. Then $\pi_{1}\left(w_{1}-w_{1}^{\prime}\right)=0$, and therefore $w_{1}-w_{1}^{\prime} \in K_{1}(\Lambda)$. Similarly $w_{2}-w_{2}^{\prime} \in K_{2}(\Lambda)$ and $\pi_{2}\left(w_{2}\right)=\pi_{2}\left(w_{2}^{\prime}\right)$ showing $\Theta$ is well defined. It is an isomorphism, because the map $\theta$, defined by reversing the roles of 1 and 2 , is an inverse map.

We now show that $Q$ is a complete invariant of the action of $L$ on $\mathcal{B}_{e} \operatorname{GL}(V)^{0}$.

Theorem 2.4. Let $\Lambda, \Lambda^{\prime}$ be $\mathcal{O}$-lattices. Then $\Lambda$ and $\Lambda^{\prime}$ are in the same $\operatorname{GL}\left(W_{1}\right) \times$ $\mathrm{GL}\left(W_{2}\right)$ orbit if and only if $Q(\Lambda)=Q\left(\Lambda^{\prime}\right)$.

Proof. The class $Q(\Lambda)$ is a $\mathrm{GL}\left(W_{1}\right) \times \mathrm{GL}\left(W_{2}\right)$-invariant since each factor of $\mathrm{GL}\left(W_{i}\right)$ commutes with the projection map $p_{i}$. We must show that if $Q(\Lambda)=$ $Q\left(\Lambda^{\prime}\right)$ then there is a $g \in \mathrm{GL}\left(W_{1}\right) \times \mathrm{GL}\left(W_{2}\right)$ so that $\Lambda=g \Lambda^{\prime}$.

We will need $g_{1} \in \mathrm{GL}\left(W_{1}\right)$ and $g_{2} \in \mathrm{GL}\left(W_{2}\right)$ so that $g_{i} P_{i}\left(\Lambda^{\prime}\right)=P_{i}(\Lambda)$ and $g_{i} K_{i}\left(\Lambda^{\prime}\right)=K_{i}(\Lambda)$ for $i=1,2$. There are certainly $g_{i} \in \operatorname{GL}\left(W_{i}\right)$ so that 


We use the basis for $V$ of the previous section to identify GL $(V)$ with $\mathrm{GL}_{n}(F)$. We will also let $K=Z\left(\mathrm{GL}_{n}(F)\right) \mathrm{GL}_{n}(\mathcal{O})$. Then $N \cong M_{n_{1} \times n_{2}}(F)$, the $n_{1} \times n_{2}$ matrices embedded in $\mathrm{GL}_{n}(F)$ as follows:

$$
\begin{aligned}
u: M_{n_{1} \times n_{2}}(F) & \rightarrow N \\
B & \mapsto\left(\begin{array}{cc}
I_{n_{1}} & B \\
0 & I_{n_{2}}
\end{array}\right) .
\end{aligned}
$$

Let $\alpha \in A^{n_{1}}$ and define $m^{\alpha} \in M_{n_{1} \times n_{2}}$ as follows:

$$
\left[m^{\alpha}\right]_{i j}= \begin{cases}\varpi^{-\alpha_{i}} & \text { if } i=j \in\left\{1, \ldots, n_{1}\right\} \\ 0 & \text { else. }\end{cases}
$$

Now let $n^{\alpha}=u\left(m^{\alpha}\right)$. Then we have the following proposition.

Proposition 2.9. We may write $\mathrm{GL}_{n}(F)$ as a disjoint union

$$
\mathrm{GL}_{n}(F)=\coprod_{\alpha \in A^{n_{1}}} L n^{\alpha} K
$$

Proof. Let $\alpha \in A^{n_{1}}$, and define $l^{\alpha}$ to be the linear transformation that sends $e_{i}$ to $e_{i}$ and $f_{i}$ to $\varpi^{-\alpha_{i}} f_{i}$ for $1 \leq i \leq n_{1}$, and $f_{j}$ to $f_{j}$ for $n_{1}+1 \leq j \leq n_{2}$. Note that $l^{\alpha} \in L$.

Let $\Lambda=\operatorname{span}_{\mathcal{O}}\left(e_{1} \ldots, e_{n_{1}}, f_{1}, \ldots, f_{n_{2}}\right)$, and notice that $K$ stabilizes $[\Lambda]$. Furthermore, we have $l^{\alpha} n^{\alpha}(\Lambda)=\Lambda^{\alpha}$.

This double coset decomposition is in no way canonical, although it has some nice properties. All the $n^{\alpha}$ are supported on the span of root groups $U^{i, i+n_{1}}$ for $1 \leq i \leq n_{1}$, with the roots taken with respect to the diagonal torus. In fact, these root group form a set of maximally mutually orthogonal root groups in $N$. Any such set of root groups can be a support of coset representatives. This can easily be seen by having $W_{n_{i}}$ the Weyl groups of $\mathrm{GL}_{n_{i}}$ act on the $n^{\alpha}$. This leads to the following conjecture for more general groups.

Conjecture 2.10. Let $G$ be a reductive group over $F$ and $P$ a parabolic subgroup with $P=L N$, and assume $N$ is abelian. Let $K$ be a maximal open, bounded subgroup of $G$. Then there is a discrete subset $N^{\prime} \subset N$ and a maximally mutually orthogonal set of root groups $U^{\alpha}<N$ so that:

1. each $n \in N^{\prime}$ is supported in the group generated by the $U^{\alpha}$;

2. $G=\coprod_{n \in N^{\prime}} \operatorname{Ln} Z(G) K$. 


\subsubsection{Stabilizers}

We now wish to compute stabilizers for each orbit so that we may realize the orbits as homogeneous spaces. For spherical buildings knowing the stabilizers plays a role in representation theory, for instance [3]. For Euclidean buildings this may have applications to understanding cuspidal representations.

Fix a $\Lambda$ and let $S_{i}=\operatorname{stab}\left(P_{i}(\Lambda)\right) \cap \operatorname{stab}\left(K_{i}(\Lambda)\right)$. Furthermore, let

$$
T_{i}=\left\{I+A \mid A \in \operatorname{End}\left(W_{i}\right) \text { and } A\left(P_{i}(\Lambda)\right) \subset K_{i}(\Lambda)\right\} \cap S_{i} .
$$

Then $T_{i} \triangleleft S_{i}$ and $S_{i} / T_{i} \cong \operatorname{GL}\left(Q_{i}(\Lambda)\right)$ by Lemma 2.1. Let

$$
\overline{S_{\Lambda}}=\left\{\left(h_{1}, \Theta_{\Lambda}^{*}\left(h_{1}\right)\right) \mid h_{1} \in \operatorname{GL}\left(Q_{1}(\Lambda)\right)\right\} \subset\left(\operatorname{GL}\left(Q_{1}(\Lambda)\right) \times \operatorname{GL}\left(Q_{2}(\Lambda)\right)\right.
$$

where $\Theta_{\Lambda}^{*}$ is the isomorphism induced on $\operatorname{GL}\left(Q_{1}(\Lambda)\right)$ from the isomorphism $\Theta_{\Lambda}: Q_{1}(\Lambda) \rightarrow Q_{2}(\Lambda)$ defined in equation (7). Finally, let $S_{\Lambda}$ be the pullback of $\overline{S_{\Lambda}}$ in $S_{1} \times S_{2}$.

Proposition 2.11. $S_{\Lambda}=\operatorname{stab}_{L}(\Lambda)$.

Proof. Let $\left(A_{1}, A_{2}\right) \in S_{\Lambda}$ with $A_{i} \in \mathrm{GL}\left(W_{i}\right)$, and let $\Lambda^{\prime}=\left(A_{1}, A_{2}\right) \cdot \Lambda$. Then because $A_{i} \in S_{i}$ we have $P_{i}(\Lambda)=P_{i}\left(\Lambda^{\prime}\right)$ and $K_{i}(\Lambda)=K_{i}\left(\Lambda^{\prime}\right)$. We now wish to show $\Theta_{\Lambda}=\Theta_{\Lambda^{\prime}}$. By the proof of Theorem 2.4 this will show that $\Lambda=\Lambda^{\prime}$. Let $B_{i}$ be the image of $A_{i}$ in $S_{i} / T_{i} \cong \mathrm{GL}\left(Q_{i}(\Lambda)\right)$, and let $v \in Q_{1}(\Lambda)$ and $v^{\prime}=B_{1}^{-1} v$. Then

$$
\begin{aligned}
\Theta_{\Lambda^{\prime}}(v) & =\Theta_{\Lambda^{\prime}}\left(B_{1} B_{1}^{-1} v\right) \\
& =B_{2} \Theta_{\Lambda}\left(B_{1}^{-1} v\right) \\
& =\Theta_{\Lambda}^{*}\left(B_{1}\right) \Theta_{\Lambda}\left(B_{1}^{-1} v\right) \\
& =\Theta_{\Lambda}\left(B_{1} \Theta_{\Lambda}^{-1}\left(\Theta_{\Lambda}\left(B_{1}^{-1} v\right)\right)\right) \\
& =\Theta_{\Lambda}(v) .
\end{aligned}
$$

Line (15) follows from the action of $\left(A_{1}, A_{2}\right)$ on $\Lambda$, line (16) comes from the fact that $B_{2}=\Theta_{\Lambda}^{*}\left(B_{1}\right)$, and line (17) is the definition of the induced map $\Theta_{\Lambda}^{*}$.

This proves $S_{\Lambda} \subset \operatorname{stab}_{L}(\Lambda)$. We now prove the other direction. Assume $\left(A_{1}, A_{2}\right) \in \operatorname{stab}_{L}(\Lambda)$, then $A_{i} \in S_{i}$. The calculation above shows that the projection of $A_{2}$ in $\operatorname{GL}\left(Q_{2}(\Lambda)\right)$ has to equal the image of $A_{1}$ in $\operatorname{GL}\left(Q_{2}(\Lambda)\right)$ under $\Theta_{\Lambda}^{*}$, proving the result.

We end this section by giving an explicit description of $S_{\Lambda^{\alpha}}$, the stabilizers of our orbit representatives. Let $\alpha \in A^{n_{1}}$, then we define $\Lambda^{\alpha}$ as in section 2.3. By 


